\title{
Avaliação da Qualidade de Comprimidos Dispensados em uma Farmácia Pública do Noroeste do Rio Grande do Sul
}

\author{
Débora Cristina Krauser ${ }^{1}$, Roberta Cattaneo Horn' ${ }^{2}$, Gabriela Bonfanti-Azzolin ${ }^{3}$, \\ Regis Augusto Norbert Deuschle ${ }^{4}$,Viviane Cecilia Kessler Nunes Deuschle ${ }^{5}$
}

\begin{abstract}
RESUMO
O controle de qualidade é um processo importante no preparo de medicamentos e deve abranger todas as etapas da produção. Desvios na qualidade de um medicamento podem causar diversos danos à saúde do paciente. O Sistema Único de Saúde (SUS) conta com distribuição gratuita de medicamentos para a população e a aquisição desses medicamentos é feita por meio de licitações. Nesse sentido, o objetivo deste trabalho foi realizar testes físico-químicos de controle de qualidade de comprimidos dispensados em uma Farmácia Pública de um município do Noroeste do Rio Grande do Sul. Foram fornecidos pela Farmácia Pública os seguintes medicamentos: Furosemida $40 \mathrm{mg}$, Enalapril $10 \mathrm{mg}$ e Propranolol $40 \mathrm{mg}$. Os testes realizados foram: análise do aspecto físico, peso médio, friabilidade, tempo de desintegração e doseamento. A Furosemida foi reprovada no teste do peso médio e aprovada nos demais testes. Já o Propranolol foi reprovado no ensaio de doseamento, encontrando-se abaixo das especificações e aprovado nos demais testes. O Enalapril apresentou conformidade em todos os testes realizados. Esses resultados demonstram que os medicamentos podem comprometer a saúde do usuário do sistema público e critérios mais rígidos de fiscalização devem ser adotados nas indústrias farmacêuticas.
\end{abstract}

Palavras-chave: Controle de qualidade. Sistema Único de Saúde. Educação continuada. Política Nacional de Medicamentos. Proposta de concorrência.

\section{QUALITY EVALUATION OF TABLETS DISPENSED IN A NORTHWESTERN RIO GRANDE DO SUL PUBLIC PHARMACY}

\section{ABSTRACT}

Quality control is an important process in drug preparation and should cover all stages of production. Deviations in the quality of a drug can cause various damages to the patient's health. The Unified Health System (SUS) has free distribution of medicines to the population and the purchase of these medicines is made through bids. In this sense, the objective of this work was to perform physical-chemical quality control tests of tablets dispensed in a Public Pharmacy in a northwestern municipality of Rio Grande do Sul. The following drugs were supplied by the Public Pharmacy: Furosemide $40 \mathrm{mg}$, Enalapril 10 $\mathrm{mg}$ and Propranolol $40 \mathrm{mg}$. The tests were: physical aspect analysis, average weight, friability, disintegration time and dosing. Furosemide failed the average weight test and passed the other tests. Propranolol, however, failed the dosing assay, being below specifications and approved in the other tests. Enalapril showed compliance in all tests performed. These results demonstrate that drugs can compromise the health of the public system user and stricter inspection criteria should be adopted in the pharmaceutical industries.

Keywords: Quality control. Unified health system. Education continuing. National drug Policy. Competitive bidding.

\footnotetext{
Farmacêutica. http://lattes.cnpq.br/5810209592595978. https://orcid.org/0000-0002-1616-8946. deborahkrauser@hotmail.com

Docente permanente no Programa de Pós-Graduação em Atenção Integral à Saúde (PPGAIS) - Universidade de Cruz Alta (Unicruz) e Universidade Regional do Noroeste do Estado do Rio Grande do Sul (Unijuí) e do Curso de Farmácia da Universidade de Cruz Alta. http://lattes.cnpq.br/4282856921907832. https:// orcid.org/0000-0001-8261-1726. rcattaneo@unicruz.edu.br

Docente permanente no Programa de Pós-Graduação em Atenção Integral à Saúde (PPGAIS) - Universidade de Cruz Alta (Unicruz) e Universidade Regional do Noroeste do Estado do Rio Grande do Sul (Unijuí) e do Curso de Farmácia da Universidade de Cruz Alta. http://lattes.cnpq.br/1516086893514285. https:// orcid.org/0000-0003-2602-6092. gbonfanti@unicruz.edu.br

${ }^{4}$ Docente do Curso de Farmácia da Universidade de Cruz Alta-RS. http://lattes.cnpq.br/1381424496248996. https://orcid.org/0000-0001-6527-4449. rdeuschle@unicruz.edu.br

Docente do Curso de Farmácia da Universidade de Cruz Alta-RS. http://lattes.cnpq.br/2302396749597987. https://orcid.org/0000-0001-6797-0376. vdeuschle@unicruz.edu.br
} 


\section{INTRODUÇÃO}

O Sistema Público de Saúde brasileiro conta com a distribuição gratuita de medicamentos para os pacientes usuários do Sistema Único de Saúde (SUS), o que garante $o$ acesso a medicamentos para todos que necessitam, principalmente em relação ao tratamento de doenças crônicas (BUENO; WEBER; MOREIRA, 2010; BIANCHIN et al., 2012).

A Relação Nacional de Medicamentos Essenciais (Rename) é uma lista utilizada pelo SUS que possui todos os insumos necessários para atender à maioria dos problemas de saúde da população. Esses medicamentos devem estar sempre disponíveis nas suas formas farmacêuticas adequadas, prontas para serem utilizadas sempre que forem necessárias. A Rename deve servir como base para a composição de listas estaduais e municipais, e ambas devem ser continuamente atualizadas, seguindo critérios epidemiológicos, de forma que os medicamentos nelas contidos possam cobrir o tratamento da maioria das doenças do país. Desta forma, a Rename tem como objetivo orientar a prescrição e a aquisição de medicamentos com o intuito de reduzir os custos (BRASIL, 2010; ALMEIDA; ANDRADE, 2014; ROSA; CAVALCANTE; SILVA, 2018).

A aquisição de medicamentos pelo SUS faz parte da Gestão da Assistência Farmacêutica e é uma de suas principais atividades. $O$ processo de aquisição ocorre da seguinte forma: primeiramente os medicamentos são selecionados (o quê e quanto comprar), levando-se em consideração os medicamentos listados na Rename e Relação Municipal de Medicamentos Essenciais (Remume), definindo-se a quantidade a ser comprada baseada no histórico de uso e na disponibilidade de recursos financeiros. A partir disso é aberta uma licitação, quando se busca a proposta mais vantajosa, ou seja, em grande quantidade e preço baixo. Depois de homologados os resultados do processo licitatório, a compra dos medicamentos é realizada, e a distribuição é feita periodicamente de acordo com o estoque da farmácia (BRASIL, 2006a; ALMEIDA; ANDRADE, 2014; BERMUDEZ et al., 2018).

Na maioria das vezes, entretanto, é levado em consideração somente o critério de valor mais baixo, sendo deixada de lado a qualidade dos medicamentos adquiridos, o que pode gerar riscos à saúde do usuário, não adesão à terapia, doses abaixo das especificações, reações adversas e até mesmo toxicidade (BIANCHIN et al., 2012; MERISIO et al., 2012).
Em razão de todos esses riscos, é de extrema importância a realização de um extenso controle de qualidade nas indústrias farmacêuticas, pois isso irá garantir a segurança e a eficácia dos medicamentos, posto que ambas irão refletir diretamente na saúde do consumidor. $O$ controle de qualidade é um dos processos mais importantes na fabricação de um medicamento, uma vez que abrange todas as etapas de desenvolvimento e produção do mesmo. Para tanto, são feitos ensaios prévios de pureza e identificação da matéria-prima, com o objetivo de evitar o uso de um produto de baixa qualidade. Durante as demais etapas são realizados ensaios de controle em processo e de controle de qualidade do produto acabado, para verificar a dose exata do fármaco na forma farmacêutica e conferir se a embalagem garante a estabilidade do medicamento (PONTES, 2009; BIANCHIN et al., 2012; ROCHA; GALENDE, 2014; MESSA et al., 2014).

Neste contexto, foi objetivo deste trabalho avaliar a qualidade dos medicamentos Furosemida, Enalapril e Propranolol dispensados por uma farmácia pública localizada no Noroeste do Estado do Rio Grande do Sul.

\section{MÉTODOS}

Primeiramente foi enviada uma Carta de Autorização à Farmácia para obter acesso aos medicamentos dispensados e garantir o sigilo dos pesquisadores em relação aos dados obtidos. Os medicamentos foram selecionados levando-se em consideração a maior saída e conforme disponibilidade de materiais para a realização das análises.

Os ensaios nos comprimidos foram realizados conforme disponibilidade de materiais na universidade e seguiu a metodologia descrita na Farmacopeia Brasileira (BRASIL, 2010), como segue:

\section{Aspecto Físico}

Os comprimidos foram analisados quanto à presença de rachaduras e quebras, uniformidade de cor, integridade e legibilidade da embalagem, comprimidos faltantes e qualquer outra irregularidade.

\section{Peso Médio}

Pesou-se individualmente 20 comprimidos e, após, calculou-se a média aritmética e os limites de variação. Admite-se uma variação de $\pm 10 \%$ para comprimidos com peso médio de até $80 \mathrm{mg} \mathrm{e} \pm 7,5 \%$ para comprimidos com peso médio acima de 80 e até 250 mg. Ainda, de acordo com a Farmacopeia Brasileira 
(BRASIL, 2010), não são toleradas mais do que duas unidades fora dos limites especificados, e nenhuma unidade, em relação ao peso médio, deve estar acima ou abaixo do dobro das porcentagens indicadas.

\section{Friabilidade}

Para este teste pesou-se 10 comprimidos que, posteriormente, foram levados ao friabilômetro (Nova Ética, modelo 300/1) por 5 minutos, em velocidade de rotação 25 rotações por minuto (rpm). Após, pesou-se os comprimidos novamente. São aceitos os comprimidos com perdas iguais ou inferiores a $1,5 \%$ do seu peso.

\section{Desintegração}

Foram colocados 6 comprimidos em cada cesta do desintegrador (Nova Ética modelo 301/AC), utilizando a água em temperatura de $37^{\circ} \mathrm{C}$ como líquido de imersão. $O$ tempo limite para que ocorra a desintegração é de 30 minutos.

\section{Doseamento}

A metodologia usada para a determinação do doseamento dos comprimidos de Furosemida, Enalapril e Propranolol foi realizada por meio de espectrofotometria na região do UV. Todas as análises foram realizadas em triplicata. Primeiramente foram pesados e triturados 20 comprimidos de Furosemida, Enalapril e Propranolol, individualmente.

Transferiu-se, analiticamente, o equivalente a $200 \mathrm{mg}$ de Furosemida para um balão volumétrico de $500 \mathrm{~mL}$. Adicionou-se cerca de $300 \mathrm{~mL}$ de hidróxido de sódio $0,1 \mathrm{M}$, agitou-se por 10 minutos e, em segui$\mathrm{da}$, completou-se o volume com o mesmo solvente. A amostra foi filtrada e, desta, retirada uma alíquota de $5 \mathrm{~mL}$, que foram transferidas para balões volumétricos de $250 \mathrm{~mL}$, completando-se os volumes com o mesmo solvente. Da mesma forma, foi preparado o padrão nas mesmas condições e concentração das amostras. Obteve-se uma concentração final de $8 \mu \mathrm{g} / \mathrm{mL}$. As leituras foram realizadas em $271 \mathrm{~nm}$, utilizando-se hidróxido de sódio 0,1 M como branco.

Para a determinação do teor de Enalapril pesou-se o equivalente a $10 \mathrm{mg}$ de Maleato de Enalapril em um balão volumétrico de $25 \mathrm{~mL}$, que foi diluído com ácido clorídrico $0,1 \mathrm{~N}$ até uma concentração de 20 $\mu \mathrm{g} / \mathrm{mL}$. Uma solução padrão foi preparada nas mesmas condições das amostras. As leituras foram realizadas em $202 \mathrm{~nm}$, utilizando-se ácido clorídrico 0,1 N como branco.
Para a determinação do teor do Propranolol, pesou-se quantidade do pó equivalente a $20,00 \mathrm{mg}$ de Cloridrato de Propranolol e transferiu-se para um balão volumétrico de $100 \mathrm{~mL}$. Adicionou-se $20 \mathrm{~mL}$ de água destilada e $50 \mathrm{~mL}$ de metanol, sob agitação, por 10 minutos. Após, completou-se o volume com metanol. A amostra foi filtrada e transferida uma alíquota de $10 \mathrm{~mL}$ para balão volumétrico de $50 \mathrm{~mL}$, completando-se o volume com o mesmo solvente. Foi preparada uma solução padrão nas mesmas condições da amostra. A concentração final foi de $0,04 \mathrm{mg} / \mathrm{mL}$. As leituras foram realizadas em $290 \mathrm{~nm}$, utilizando-se o metanol como branco.

Determinou-se o teor de substância ativa em porcentagem, empregando-se a Equação de Lambert-Beer:

\section{Ca=Absa /Absp X cp}

Em que:

Ca: concentração da amostra

Absa: absorbância da amostra

Absp: absorbância do padrão

$C p$ : concentração do padrão

\section{Análise dos dados}

A análise dos resultados foi realizada por meio do programa Microsoft Excel 2010.

\section{RESULTADOS E DISCUSSÃO}

Para que a terapia medicamentosa tenha resultados satisfatórios é necessário que, além da dispensação dos medicamentos e adesão do paciente ao tratamento, o medicamento cumpra todas as especificações de qualidade exigidas, garantindo a eficácia e a segurança. Dessa forma, o controle de qualidade é realizado para assegurar que esses medicamentos tenham as mesmas características em todos os lotes produzidos (TAVARES et al., 2013; CÂMARA et al., 2013; TAVARES et al., 2016).

Quanto ao aspecto, os comprimidos devem estar íntegros, sua superfície deve ser homogênea, lisa e brilhante, a cor deve estar preservada, não possuindo falhas, fissuras e contaminações (SILVA et al., 2009). Dessa forma, os comprimidos de Furosemida, Enalapril e Propranolol apresentaram conformidade com as especificações em relação à análise do aspecto (Tabela 1). 
Tabela 1 - Avaliação do aspecto e determinação dos parâmetros de peso para os comprimidos de Furosemida, Enalapril e Propranolol

\begin{tabular}{cccccc}
\hline Medicamento & Aspecto & $\begin{array}{c}\text { Peso Médio }(\mathbf{m g}) \\
\pm \mathrm{DP} *\end{array}$ & $\begin{array}{c}\text { Coeficiente de } \\
\text { Variação }(\%)\end{array}$ & $\begin{array}{c}\text { Limite inferior } \\
(\mathbf{m g})\end{array}$ & $\begin{array}{c}\text { Limite superior } \\
(\mathbf{m g})\end{array}$ \\
\hline Furosemida & CONFORME & $158 \pm 0,013$ & 8,6 & 146 & 169 \\
Enalapril & CONFORME & $78 \pm 0,0006$ & 0,84 & 70 & 86 \\
Propranolol & CONFORME & $150 \pm 0,005$ & 3,4 & 138 & 161 \\
\hline
\end{tabular}

Fonte: Dados da pesquisa.

De todos os processos envolvidos na produção de comprimidos, o mais importante é a etapa da compressão, pois é esse processo que controla as variáveis físicas de peso, dureza, friabilidade e desintegração. Falhas nessa etapa levam a desvios de qualidade (BIANCHIN et al., 2012; MESSA et al., 2014).

O controle do processo de compressão é de extrema importância para o ajuste do peso dos comprimidos neste, pois desvios nesse parâmetro podem indicar excesso ou falta de princípio ativo, prejudicando a terapia, levando a efeitos tóxicos ou não atingindo a concentração sérica necessária para desencadear efeito terapêutico. Alterações nas quantidades de excipientes também são prejudiciais, pois os mesmos são os responsáveis pela consistência e resistência do comprimido (RODRIGUES; ÁLVARES, 2017). Os resultados de peso médio obtidos para os comprimidos de Furosemida, Enalapril e Propranolol estão demonstrados na Tabela1.

De acordo com a Farmacopeia Brasileira (BRASIL, 2010), é aceita uma variação de $\pm 10 \%$ para comprimidos com peso médio de até $80 \mathrm{mg}$ e $\pm 7,5 \%$ para comprimidos com peso médio acima de 80 e até 250 $\mathrm{mg}$. Não se tolera mais do que duas unidades fora dos limites especificados e nenhuma unidade, em relação ao peso médio, pode estar acima ou abaixo do dobro das porcentagens indicadas.

Durante a análise verificou-se que dois comprimidos de Furosemida ficaram abaixo do limite mínimo e quatro comprimidos ficaram acima do limite máximo. Desta forma, a Furosemida apresentou seis unidades fora dos limites inferior e superior, preconizados na Farmacopeia Brasileira, sendo reprovada nesse teste (BRASIL, 2010). Além disso, apresentou o maior coeficiente de variação $(8,6 \%)$ em relação aos demais comprimidos (Tabela 1). Os comprimidos de Enalapril e Propranolol não apresentaram nenhuma unidade fora dos limites especificados e foram aprovados no teste.

O teste de friabilidade é um dos testes que avalia a resistência mecânica dos comprimidos. Durante a produção, embalagem, armazenamento, transporte e manuseio pelo paciente, os comprimidos estão sujeitos a sofrer choques mecânicos. Dessa forma, os mesmos precisam ser resistentes, apresentando uma baixa friabilidade, a fim de evitar a formação de rachaduras, fissuras e ocorrência de esfarelamentos (BRAGA; ROCHA; SILVA, 2015). Comprimidos muito friáveis levam a prejuízos na terapia, pois ocorre perda de parte do princípio ativo, comprometendo a eficácia do medicamento (PONTES, 2009; OLIVEIRA; CAMPOS, 2014). Os resultados de friabilidade obtidos para os comprimidos analisados estão demonstrados na Tabela 2.

Tabela 2 - Determinação da friabilidade dos comprimidos de Furosemida, Enalapril e Propranolol

\begin{tabular}{cccc}
\hline Medicamento & $\begin{array}{c}\text { Peso Inicial } \\
(\mathrm{g})\end{array}$ & $\begin{array}{c}\text { Peso Final } \\
(\mathrm{g})\end{array}$ & $\%$ Perda \\
\hline Furosemida & 1,63 & 1,63 & 0 \\
Enalapril & 0,786 & 0,783 & 0,38 \\
Propranolol & 1,50 & 1,50 & 0 \\
\hline
\end{tabular}

Fonte: Dados da pesquisa.

A Farmacopeia Brasileira (BRASIL, 2010) considera aceitável uma perda igual ou inferior a $1,5 \%$ de massa dos comprimidos. Durante o teste, os comprimidos de Furosemida e Propranolol não apresentaram perda. Os comprimidos de Enalapril apresentaram uma perda de $0,38 \%$, entretanto encontra-se dentro do limite estipulado. Nenhum comprimido apresentou rachadura, esfarelamento ou qualquer alteração em seu aspecto após ser retirado do friabilômetro. Dessa forma, pode-se afirmar que os comprimidos possuem uma resistência adequada e não irão sofrer perdas significativas durante o manuseio e, consequentemente, não irão prejudicar a terapia.

A desintegração de um comprimido é de extrema importância para que o fármaco esteja disponível e exerça sua ação farmacológica. O comprimido precisa desintegrar-se em pequenas partículas, aumentando a superfície de contato com o meio, facilitando sua absorção pelo organismo, isto é, a desintegração de um comprimido está diretamente ligada à sua biodisponibilidade e ação terapêutica (PONTES, 2009). Um comprimido que desintegra fora das especificações poderá ser absorvido de forma inadequada e apre- 
sentar problemas referentes à biodisponibilidade, não atingindo a concentração sérica necessária para que ocorra a ação farmacológica (MESSA et al., 2014; OLIVEIRA; CAMPOS, 2014).

Para o teste de desintegração, o tempo limite para que todos os comprimidos desintegrem é de 30 minutos, salvo exceções mencionadas na monografia de cada medicamento. O comprimido é considerado desintegrado quando nenhum resíduo permanece na tela da cesta após terminar o tempo, salvos resíduos insolúveis que compõem o revestimento. São consideradas desintegradas as unidades que formarem uma massa pastosa sem núcleo palpável (BRASIL, 2010). Os resultados do tempo de desintegração obtidos para os comprimidos em testes estão demonstrados na Tabela 3.

Tabela 3 - Tempo de Desintegração e doseamento dos comprimidos de Furosemida, Enalapril e Propranolol

\begin{tabular}{ccc}
\hline Amostra & $\begin{array}{c}\text { Tempo de } \\
\text { desintegração } \\
\text { (minutos) }\end{array}$ & Teor (\%) \pm DP* \\
\hline Furosemida & 2 minutos & $98,2 \pm 3,2$ \\
Enalapril & 9 minutos & $102,75 \pm 5,5$ \\
Propranolol & 22 segundos & $85 \pm 6,0$ \\
\hline
\end{tabular}

Fonte: Dados da pesquisa. *DP: Desvio padrão.

Todos os comprimidos desintegraram dentro do tempo limite especificado e, dessa forma, pressupõe-se que ocorrerá uma dissolução adequada para que os fármacos sejam devidamente absorvidos e para que ocorra a ação farmacológica.

Em relação ao teor de princípio ativo, observa-se que a Furosemida e o Enalapril ficaram dentro dos limites estabelecidos pela Farmacopeia Brasileira (mínimo $90 \%$ e máximo $110 \%$ do valor rotulado) (BRASIL, 2010). Os comprimidos de Propranolol, contudo, apresentaram valores abaixo do especificado (Tabela 3).

O teor do fármaco declarado abaixo ou acima dos valores especificados consiste em comprometimento da terapia e risco para a saúde do usuário. Quando o teor está abaixo da especificação, o fármaco poderá não apresentar o efeito terapêutico necessário. As amostras de medicamentos analisadas neste estudo não apresentaram valores de teor acima do especificado. Teor acima do recomendado, no entanto, também constitui em risco para a saúde pela possibilidade de ocorrência de efeitos adversos e intoxicações (BIANCHIN et al., 2012; RODRIGUES; ÁLVARES, 2017; DUROVISKI; OLIVEIRA, 2018).
A variabilidade de medicamentos oferecidos pelo mercado nacional é muito grande, e a qualidade desses medicamentos é bastante discutida e alvo de estudos. Bianchin et al. (2012) avaliaram a qualidade de comprimidos de Propranolol e Enalapril dispensados pelo sistema público em um município do Sul do Brasil e demonstraram que três amostras de Propranolol e duas de Enalapril encontravam-se fora das especificações, sendo reprovadas nos testes. Uma amostra de Enalapril apresentou teor de $88,16 \% \pm 0,001 \%$, corroborando nossos resultados para os comprimidos de Propranolol $(85 \% \pm 6,0)$. Messa et al. (2014) avaliaram a qualidade de comprimidos de Hidroclorotiazida (referência, similar e genérico) e os resultados demonstraram que os comprimidos similares foram reprovados no ensaio de desintegração. Outro estudo verificou a equivalência farmacêutica dos medicamentos Captopril e Cloridrato de Propranolol comercializados no programa Farmácia Popular do Brasil, quando foram detectadas não conformidades no teste de dissolução do Captopril, que apresentou uma extensão de dissolução inferior quando comparado com o medicamento de referência e genérico (PONTES, 2009). Em nosso estudo não foi realizado o teste de dissolução. Observa-se, entretanto, que há variações em relação às especificações para os medicamentos, verificadas em diferentes estudos, tornando-se uma questão preocupante, principalmente para o setor público, que utiliza o processo licitatório para aquisição dos medicamentos.

O controle de qualidade pode ser definido como um conjunto de especificações que precisam ser atendidas para tornar um produto apto para o consumo. $\mathrm{Na}$ indústria de medicamentos esse processo é imprescindível e deve abranger todas as etapas da produção. Os desvios de qualidade na produção de medicamentos apresentam grandes riscos para a segurança do paciente, podendo levar ao agravamento de uma enfermidade que não está sendo devidamente tratada, como também ao desenvolvimento de novos problemas de saúde, sendo eles pela falta de uma ação eficaz do medicamento ou por atingir concentrações tóxicas e perigosas (BUENO; WEBER; MOREIRA, 2010; BERNARDES; MUELLER; GEBARA, 2010; SILVA et al., 2014).

A Política Nacional de Medicamentos define assistência farmacêutica como o "conjunto de atividades relacionadas ao medicamento, com a finalidade de apoiar as ações em saúde destinadas a uma determinada comunidade". Dessa forma, a assistência farmacêutica faz parte do cuidado à saúde, seja de forma coletiva ou individual, em que o acesso aos medica- 
mentos deve ser garantido por intermédio de seu uso racional. Ainda, as ações relacionadas ao medicamento devem ser feitas em todas as suas dimensões, mas com ênfase no paciente e na comunidade em geral por meio de medidas de promoção à saúde (BRASIL, 2006b; ARAUJO, 2008; VIEIRA, 2010; PEREIRA, 2015).

Desvios da qualidade comprometem a saúde do usuário, aumentando os riscos de falha na terapia e reações adversas ou tóxicas. Como se observa, dois lotes de medicamentos analisados apresentaram problemas quanto a sua qualidade e, desta forma, enfatiza-se a necessidade de monitoramento das compras realizadas pelo setor público. Bianchin et al. (2012) ressaltam que esse processo pode ser realizado mediante controle de qualidade pela farmácia, antes de o medicamento chegar ao paciente, mas também destaca que a realização dos testes demanda tempo e laboratórios especializados com custos elevados. Desta forma, a melhor maneira de monitorar as não conformidades relacionadas aos medicamentos é por meio da farmacovigilância.

Neste contexto, a farmacovigilância deve ser incentivada como forma de obter dados sobre a qualidade dos medicamentos, como ineficácia, alterações físico-químicas e problemas com embalagens. Esses dados também são importantes para a qualificação dos fornecedores, o que pode contribuir para a definição das empresas que participarão ou não nos processos licitatórios (BIANCHIN et al., 2012; MODESTO et al., 2016)

Ao mesmo tempo sugere-se que a Vigilância Sanitária adote critérios mais rígidos de fiscalização na indústria farmacêutica brasileira, visando a uma maior qualidade dos medicamentos produzidos e garantindo que a saúde do paciente não sofra prejuízos.

\section{CONCLUSÃO}

Ao final das análises dos comprimidos dispensados por uma farmácia pública do Noroeste do Estado do Rio Grande do Sul, pode-se concluir que a Furosemida e o Propranolol apresentaram problemas de qualidade em alguns dos testes realizados, o que pode comprometer a eficácia dos mesmos. Já o Enalapril mostrou conformidade com todos os testes realizados. Como um dos objetivos deste trabalho foi contribuir para o melhoramento da aquisição de medicamentos pelo sistema público, sugere-se que a adoção de análises de controle de qualidade pode ser uma ferramenta útil para o monitoramento de diferentes lotes e para qualificar fornecedores que participam dos processos licitatórios. Ainda, ressalta-se que a atuação da Vigilância Sanitária deveria adotar critérios mais rígidos na fiscalização das indústrias de medicamentos brasileiras que participam dos processos de licitação, uma vez que vence a que apresentar o menor valor, garantindo maior qualidade dos medicamentos que chegam ao sistema público.

\section{REFERÊNCIAS}

ALMEIDA, C. C.; ANDRADE, K. V. F. Assistência farmacêutica no Sistema Único de Saúde (SUS): conceito, histórico e dispositivos legais. Rev. Saúde.com., v. 10, n. 1, p. 80-86, 2014.

ARAUJO, A. L. A. Perfil da assistência farmacêutica na atenção primária do Sistema Único de Saúde. Ciênc. Saúde Coletiva, v. 13, n. Sup, p. 611-617, 2008.

BERMUDEZ, J. A. Z. et al. Assistência farmacêutica nos 30 anos do SUS na perspectiva da integralidade. Cienc. Saúde Coletiva, v. 26, n. 3, p. 1.937-1.951, 2018.

BERNARDES, M. L. P.; MUELLER, A.; GEBARA, K. S. Desenvolvimento e controle de qualidade de cápsulas contendo captopril 25 mg. Interbio., v. 4, n. 1, p. 15-21, 2010.

BIANCHIN, M. D. et al. Avaliação de comprimidos de propranolol e enalapril distribuídos no Sistema Único de Saúde em uma cidade do sul do Brasil. Ciênc. Saúde Coletiva, v. 17, n. 2, p. 491-498, 2012.

BRAGA, R. R.; ROCHA, A. C.; SILVA, E. R. Controle de qualidade físico-químico de comprimidos de cloridrato de propranolol dispensados pelo programa farmácia popular do Brasil. Revista Perspectivas da Ciência e Tecnologia, v. 7, n. 1, p. 45-54, 2015.

BRASIL. Agência Nacional de Vigilância Sanitária. Farmacopeia Brasileira. 5. ed. Ministério da Saúde. Brasília: Anvisa, 2010.

BRASIL. Ministério da Saúde. Aquisição de medicamentos para assistência farmacêutica no SUS. Diário Oficial da União, Brasília, 2006a. (Série A. Normas e Manuais Técnicos).

BRASIL. Ministério da Saúde. Secretaria de Ciência, Tecnologia e Insumos Estratégicos. Departamento de Assistência Farmacêutica e Insumos Estratégicos. Assistência farmacêutica na atenção básica: instruções técnicas para sua organização. 2. ed. Brasília: Ministério da Saúde, 2006b.

BUENO, C. S.; WEBER, D.; MOREIRA, A. C. Avaliação da qualidade de quatro especialidades farmacêuticas contendo hidroclorotiazida. Rev. Bras. Farm., v. 91, n. 3, p. 126-132, 2010.

CÂMARA, R. P. et al. Avaliação da qualidade físico-química de comprimidos de enalapril: referência, genérico e similar, dispensados na rede pública e privada na cidade de Montes Claros-MG. Revista Conexão Ciência, v. 8, n. 2, p. 52-62, 2013.

DUROVISKI, D.; OLIVEIRA, V. B. Avaliação da equivalência farmacêutica de comprimidos de nimesulida. Visão Acadêmica, v. 19, n. 3, p. 38-45, 2018. 
MERISIO, A. et al. A aquisição de medicamentos para o Sistema Único de Saúde em municípios de pequeno porte do Estado de Santa Catarina. Rev. Bras. Farm., v. 93, n. 2, p. 173-178, 2012.

MESSA, R. V. et al. Avaliação da qualidade de comprimidos de hidroclotiazida: medicamentos de referência, genérico e similar comercializados na cidade de Dourados - MS. Interbio., v. 8, n. , p. 72-78, 2014.

MODESTO, A. C. F. et al. Reações adversas a medicamentos e farmacovigilância: conhecimentos e condutas de profissionais de saúde de um Hospital da rede Sentinela. Rev. Bras. Educ. Méd., v. 40, n. 3, p. 401-410, 2016.

OLIVEIRA, V. C. B.; CAMPOS, R. Estudo da equivalência farmacêutica de comprimidos de ibuprofeno. Cadernos da Escola de Saúde, v. 1, n. 11, p. 56-64, 2014.

PEREIRA, R. M. Planejamento, programação e aquisição: prever para prover. Organização Pan-americana da Saúde, v. 1, n. 10, p. 1-7, 2015.

PONTES, A. V. Estudo de equivalência farmacêutica dos fármacos captopril e cloridrato de propranolol comercializados no programa farmácia popular do Brasil. 2009. 144 f. Dissertação (Mestrado em Farmacologia) - Universidade Federal do Ceará, Fortaleza, CE, 2009.

ROCHA, T. G.; GALENDE, S. B. A importância do controle de qualidade na indústria farmacêutica. Revista Uningá Review, v. 20, n. 2, p. 97-103, 2014.

RODRIGUES, T. J.; ÁLVARES, A. C. M. Estudo comparativo das propriedades físico-químicas de comprimidos de cloridrato de metformina comercializados no interior de Goiás. Revista Científica Sena Aires, v. 6, n. 2, p. 126-133, 2017.

ROSA, I. S. S.; CAVALCANTE, M. S.; SILVA, C. F. S. Assistência farmacêutica no Sistema Único de Saúde. Rev. Cient. Faema, v. 9, ed. Especial, p. 519-519, 2018.

SILVA, F. E. B. et al. Desenvolvimento de comprimidos contendo fluconazol por compressão direta. Lat. Am. J. Pharm., v. 28, n. 4, p. 604-608, 2009.

SILVA, F. J. et al. Controle de qualidade físico-químico de comprimidos de ibuprofeno $300 \mathrm{mg}$. Revista Faculdade Montes Belos (FMB), v. 7, n. 1, p. 151-162, 2014.

TAVARES, N. U. L. et al. Fatores associados à baixa adesão ao tratamento medicamentoso em idosos. Rev. Saúde Pública., v. 47, n. 6, p. 1-9, 2013.

TAVARES, N. U. L. et al. Fatores associados à baixa adesão ao tratamento farmacológico de doenças crônicas no Brasil. Rev. Saúde Pública, v. 50, n. supl 2, p. 1S-11s, 2016.

VIEIRA, F. S. Assistência farmacêutica no sistema público de saúde no Brasil. Rev. Panam. Salud Publica, v. 27, n. 2, p. 149-156, 2010. 